\title{
MHD and Mixed Convection Flow of Maxwell Fluid on Heat Transfer near a Stagnation Point Flow
}

\author{
Kotha Gangadhar ${ }^{1}$, Thommaandru RangaRao ${ }^{1}$, M. J. Subhakar ${ }^{2}$ and \\ T.V.S. Sekhar \\ ${ }^{1}$ *Department of Mathematics, Acharya Nagarjuna University, Ongole, Andhra Pradesh -523001, India \\ ${ }^{1}$ Department of Mathematics, SVKP College, Markapur, Prakasam DT-523316, India \\ ${ }^{2}$ Department of Mathematics, Noble College, Machilipatnam, A.P. 521001.India. \\ ${ }^{3}$ Associate Professor, School of Basic Science (mathematics), IIT Bhubaneswar-751001
}

\begin{abstract}
The effects of thermal radiation and heat transfer of a Maxwell fluid near a mixed convection stagnation point flow over a moving surface in the presence of MHD has been studied. The governing differential equations are transformed into a set of coupled non-linear ordinary differential equations and then solved with a numerical technique using appropriate boundary conditions for various physical parameters. The numerical solution for the governing non-linear boundary value problem is based on applying the fourth-order Runge-Kutta method coupled with the shooting technique using appropriate boundary conditions for various physical parameters. The effects of various parameters like the viscosity parameter, radiation parameter, mixed convection parameter, Deborah number, magnetic parameter and Prandtl number on the velocity and temperature profiles as well as on the local skin-friction coefficient and the local Nusselt number are presented and discussed.
\end{abstract}

Keywords: Mixed convection flow, Thermal radiation, Maxwell fluid, heat transfer, MHD, Convective condition.

\section{Introduction}

The fluids like soups, shampoos, tomato paste, condensed milk, sugar solution, apple source, mud etc. cannot be described by the Newton's law of viscosity. Such fluids are known as the non-Newtonian fluids. The non-Newtonian fluids in view of their diverse rheological properties cannot be examined through one constitutive relationship between shear stress and rate of strain. Many models of non-Newtonian fluids exist. Maxwell model is one subclass of rate type fluids. This fluid model is especially useful for polymers of low molecular weight. In view of its simplicity, this fluid model has acquired special status amongst the recent workers in the field. For instance Wang and Tan [1] discussed the flow of Maxwell fluid in a porous medium. Exact solution of Helical flows of Maxwell fluid with shear stress on the boundary is addressed by Jamil and Fetecau [2]. Zierep and Fetecau [3] studied Rayleigh-Stokes problem using Maxwell fluid. Exact solution is constructed here. Numerical solution for stagnation point flow of Maxwell fluid was computed by Sadeghy et al. [4]. Megahed [5] studied the variable fluid properties and variable heat flux effects on the flow and heat transfer in a non-Newtonian Maxwell fluid over an unsteady stretching sheet with slip velocity. An exact solution for the stretching /shrinking wall problem in a viscous fluid was provided by Yao et al. [6].

The mixed convection flow occurs in several industrial and technical applications which include nuclear reactors cooled during emergency shutdown, electronics devices cooled by fans, heat exchangers placed in a low velocity environment, and solar central receivers exposed to wind currents. In the study of fluid over heated or cooled surfaces, it is customary to neglect the effect of the buoyancy forces when the flow is horizontal. However for vertical or inclined surfaces, the buoyancy force modifies the flow field and hence the heat transfer rate. Therefore, it is not possible to neglect the effect of buoyancy forces for vertical or inclined heated or cooled surfaces. In recent years, much attention has been paid to develop efficient energy systems. Many recent studies have been focused on the problem of magnetic field effect on laminar mixed convection boundary layer flow over a vertical non-linear stretching sheet [7-9].

The radiative effects have important applications in physics and engineering particularly in space technology and high temperature processes [16]. Effects of radiation have been studied by Abdul Hakeem and Sathiyanathan [17], Seddeek and Abdelmeguid [18], Mamun Molla and Anwar Hossain [19], Hayat et al. [20] studied the mixed convection radiative flow of maxwell fluid near a stagnation point with convective condition. Soid et al. [21] studied the magnetohydrodynamics boundary layer flows over a stretching surface with radiation effect and embedded in porous medium.

The heat source/sink effects in thermal convection are significant where there may exist high temperature differences between the surface (e.g. space craft body) and the ambient fluid. Heat generation is also important in the context of exothermic or endothermic chemical reaction. Tania et al [22] has investigated 
the Effects of radiation, heat generation and viscous dissipation on MHD free convection flow along a stretching sheet. Moalem [23] studied the effect of temperature dependent heat sources taking place in electrically heating on the heat transfer within a porous medium. Vajravelu and Nayfeh [24] reported on the hydro magnetic convection at a cone and a wedge in the presence of temperature dependent heat generation or absorption effects. Swati Mukhopadhyay [25] analyzes the heat transfer analysis of the unsteady flow of a Maxwell fluid over a stretching surface in the presence of a heat source/sink.

The present study contains an analysis of the effects of mixed convective flow of a Maxwell fluid over a stretching sheet by taking MHD into account. Using the similarity transformations, the governing equations have been transformed into a set of ordinary differential equations, which are nonlinear and cannot be solved analytically, therefore, fourth order Runge-Kutta method along with shooting technique has been used for solving it. The results for velocity and temperature functions are carried out for the wide range of important parameters namely, magnetic parameter, viscosity parameter, thermal conductivity parameter and radiation parameter. The skin friction and rate of heat transfer have also been computed.

\section{Mathematical Formulation}

Let us consider the two-dimensional mixed convection stagnation point flow of an incompressible and radiative Maxwell fluid near a stretched surface. The flow is in the region $y>0$ and is subjected to a nonuniform magnetic field applied normally to the surface, $B_{0}$ is the initial strength of the magnetic field. It is assumed that the magnetic Reynolds number is very small and as there is no electric field, the electric field due to polarization of charges is neglected. Under these assumptions along with the Boussinesq and boundary layer approximations, the system of equations, which models the flow is given by

Continuity equation

$\frac{\partial u}{\partial x}+\frac{\partial v}{\partial y}=0$

Linear momentum equation

$u \frac{\partial u}{\partial x}+v \frac{\partial u}{\partial y}+\lambda_{1}\left[u^{2} \frac{\partial^{2} u}{\partial x^{2}}+v^{2} \frac{\partial^{2} u}{\partial y^{2}}+2 u v \frac{\partial^{2} u}{\partial x \partial y}\right]$
$=u_{e} \frac{d u_{e}}{d x}+v \frac{\partial^{2} u}{\partial y^{2}}-\frac{\sigma B_{0}^{2}}{\rho} u+g \beta_{T}\left(T-T_{\infty}\right)$

Energy equation

$u \frac{\partial T}{\partial x}+v \frac{\partial T}{\partial y}=\frac{1}{\rho c_{p}} \frac{\partial}{\partial y}\left(\kappa \frac{\partial T}{\partial y}\right)-\frac{\partial q_{r}}{\partial y}$

The boundary conditions for the velocity, temperature and concentration fields are

$u=u_{w}(x)=c x, v=0,-k \frac{\partial T}{\partial y}=h\left(T_{f}-T\right)$ at $y=0$

$u=u_{e}(x)=a x, T \rightarrow T_{\infty}$ as $y \rightarrow \infty$

where $\mathrm{u}$ and $\mathrm{v}$ are the velocity components along $\mathrm{x}$ and $\mathrm{y}$ directions, respectively, $\rho$ is the fluid density, $T$ is the temperature of the fluid, $\lambda_{1}$ is the relaxation time, $c_{p}$ is the specific heat at constant pressure, $\mu$ is the fluid viscosity, $k$ is the fluid thermal conductivity, $T_{\infty}$ is the free stream temperature and $T_{f}$ is the convective fluid temperature.

By using the Rosseland approximation the radiative heat flux $q_{r}$ is given by

$q_{r}=-\frac{4 \sigma *}{3 k *} \frac{\partial T^{4}}{\partial y}$

Where $\sigma *$ is the Stefan -Boltzmann constant and $k^{*}$ is the mean absorption coefficient. It should be noted that by using the Rosseland approximation, the present analysis is limited to optically thick fluids. If temperature differences within the flow are significantly small, then equation [2.5] can be linearised by expanding $T^{4}$ into the Taylor series about $T_{\infty}$, which after neglect higher order terms takes the form:

$T^{4} \cong 4 T_{\infty}^{3} T-3 T_{\infty}^{4}$

In view of equations (2.5) and (2.6), eqn. (2.3) reduces to

$u \frac{\partial T}{\partial x}+v \frac{\partial T}{\partial y}=\alpha \frac{\partial^{2} T}{\partial y^{2}}+\frac{16 \sigma * T_{\infty}^{3}}{3 k * \rho c_{p}} \frac{\partial^{2} T}{\partial y}$ 
The continuity equation (2.1) is satisfied by the Cauchy Riemann equations

$u=\frac{\partial \psi}{\partial y}$ and $v=-\frac{\partial \psi}{\partial x}$

where $\psi(x, y)$ is the stream function.

In order to transform equations (2.2) and (2.7) into a set of ordinary differential equations, the following similarity transformations and dimensionless variables are introduced.

$\psi=\sqrt{v c} x f(\eta), \eta=y \sqrt{\frac{c}{v}}, u=c x f^{\prime}(\eta), v=-\sqrt{c v} f(\eta)$

$\theta(\eta)=\frac{T-T_{\infty}}{T_{f}-T_{\infty}}, \alpha=\frac{a}{c}, \beta=\lambda_{1} c, R=\frac{4 \sigma * T_{\infty}^{3}}{k * k \rho c_{p}}, \lambda=\frac{G r_{x}}{\operatorname{Re}_{x}^{2}}$

$M=\frac{\sigma B_{0}^{2}}{\rho c}, G r_{x}=\frac{g \beta_{T}\left(T_{w}-T_{\infty}\right) x^{3}}{v^{2}}, \operatorname{Pr}=\frac{v}{\alpha}$

where $f(\eta)$ is the dimensionless stream function, $\theta$ - dimensionless temperature, $\eta$ - similarity variable, $M$ Magnetic parameter, $a$ and $c$ are constants, $\beta$ - Deborah number, $\lambda$ - mixed convection parameter, $\alpha$-ratio of rate constant, $G r_{x}$ - Grashof number, $\mathrm{Re}_{x}$ - Reynolds number, $R$ - radiation parameter, $\operatorname{Pr}$ - Prandtl number.

In view of Equations (2.8) - (2.9), the Equations (2.2) and (2.7) transform into

$$
\begin{aligned}
& f^{\prime \prime \prime}+f f^{\prime \prime}-f^{\prime 2}+\alpha^{2}-M f^{\prime}-\beta\left(f^{2} f^{\prime \prime \prime}-2 f f^{\prime} f^{\prime \prime}\right)+\lambda \theta=0 \\
& \left(1+\frac{4}{3} R\right) \theta^{\prime \prime}+\operatorname{Pr} f \theta^{\prime}=0
\end{aligned}
$$

The corresponding boundary conditions are:

$$
\begin{aligned}
& f(0)=0, f^{\prime}(0)=1, \theta^{\prime}(0)=-\gamma(1-\theta(0)) \\
& f^{\prime}(\infty)=\alpha, \theta(\infty)=0
\end{aligned}
$$

where the primes denote differentiation with respect to $\eta$

The physical quantities of interest are the skin friction coefficient $C_{f}$, the local Nusselt number $\mathrm{Nu}$ which are defined as

$$
C_{f}=-2 \operatorname{Re}_{x}^{-1 / 2} f^{\prime \prime}(0), N u=-\operatorname{Re}_{x}^{1 / 2} \theta^{\prime}(0)
$$

\section{Solution of the problem}

The set of coupled non-linear governing boundary layer equations (2.10) and (2.11) together with the boundary conditions (2.12) are solved numerically by using Runge-Kutta fourth order technique along with shooting method. First of all, higher order non-linear differential Equations (2.10) and (2.11) are converted into simultaneous linear differential equations of first order and they are further transformed into initial value problem by applying the shooting technique (Jain et al.[22]). The resultant initial value problem is solved by employing Runge-Kutta fourth order technique. The step size $\Delta \eta=0.05$ is used to obtain the numerical solution with five decimal place accuracy as the criterion of convergence. From the process of numerical computation, the skin-friction coefficient and Nusselt number, which are respectively proportional to $f^{\prime \prime}(0)$ and $-\theta^{\prime}(0)$, are also sorted out and their numerical values are presented in a tabular form.

\section{Results and Discussion}

The governing equations (2.10) - (2.11) subject to the boundary conditions (2.12) are integrated as described in section 3. In order to get a clear insight of the physical problem, the velocity and temperature have been discussed by assigning numerical values to the parameters encountered in the problem. The effects of various parameters on velocity profiles in the boundary layer are depicted in Figs. 1-7. The effects of various parameters on temperature profiles in the boundary layer are depicted in Figs. 8-13.

Fig. 1 shows the variation of the velocity with the viscosity parameter $(\alpha)$. It is noticed that the velocity thickness increases with an increase in the viscosity parameter. Fig. 2 shows the variation of the velocity with the Deborah number $(\beta)$. It is noticed that the velocity thickness decreases with an increase in the Deborah number. Fig. 3 illustrates the effect of the convective parameter $(\gamma)$ on the velocity field. It is seen that as the convective parameter increases, the velocity field increases. Fig. 4 shows the dimensionless velocity profiles for different values of magnetic parameter $(M)$. It is seen that, as expected, the velocity increases with an increase of 
magnetic parameter. The magnetic parameter is found to retard the velocity at all points of the flow field. It is because that the application of transverse magnetic field will result in a resistive type force (Lorentz force) similar to drag force which tends to resist the fluid flow and thus reducing its velocity. Also, the boundary layer thickness increases with an increase in the magnetic parameter. Fig.5 illustrates the effect of the mixed convection parameter $(\lambda)$ on the velocity field. It is seen that as the mixed convection parameter increases, the velocity field increases. Fig. 6 shows the variation of the thermal boundary-layer with the Prandtl number $(P r)$. It is noticed that the thermal boundary layer thickness decreases with an increase in the Prandtl number. Fig. 7 shows the variation of the velocity with the radiation parameter $(R)$. It is noticed that the velocity thickness increases with an increase in the radiation parameter.

Fig. 8 depicts the thermal boundary-layer with the viscosity parameter. It is noticed that the thermal boundary layer thickness decreases with an increase in the viscosity parameter. Fig. 9 depicts the thermal boundary-layer with the convective parameter. It is noticed that the thermal boundary layer thickness increases with an increase in the convective parameter. Fig. 10 shows the variation of the thermal boundary-layer with the magnetic parameter. It is observed that the thermal boundary layer thickness increases with an increase in the magnetic parameter. Fig.11 illustrates the effect of the mixed convection parameter on the temperature. It is noticed that as the mixed convection parameter increases, the temperature decreases. Fig. 12 shows the variation of the thermal boundary-layer with the Prandtl number. It is noticed that the thermal boundary layer thickness decreases with an increase in the Prandtl number. Fig. 13 shows the variation of the thermal boundary-layer with the radiation parameter. It is observed that the thermal boundary layer thickness increases with an increase in the radiation parameter.

Table 1 show the variation of the skin friction and Nusselt number with for different values of $\alpha, \beta, \gamma$, $M, \lambda, R$ and $P r$. It is noticed that the skin friction increases where as Nusselt number decrease with an increase in the Deborah number or Magnetic parameter or radiation parameter. It is found that the skin friction decreases where as Nusselt number increase with an increase in the viscosity parameter or convective parameter 0r mixed convective parameter. It is observed that both the skin friction and Nusselt number increases with an increase in the Prandtl number. The correctness of the present numerical method is checked with the results obtained by Pop et al. [27], Mahapatra and Gupta [28] and Hayat et al. [20] for the values of Skin friction coefficient in the limiting condition. Thus, it is seen from Table 2.

\section{Conclusions}

The effects of thermal radiation and heat transfer of a Maxwell fluid near a mixed convection stagnation point flow over a moving surface in the presence of MHD has been studied. The governing equations are approximated to a system of non-linear ordinary differential equations by similarity transformation. Numerical calculations are carried out for various values of the dimensionless parameters of the problem. It has been found that

1. The velocity decreases as well as temperature increases with an increase in the magnetic parameter.

2. The velocity and temperature decreases with an increase in the Prandtl parameter.

3. The skin friction reduces the Prandtl number and increases with the Magnetic parameter or radiation parameter.

4. The Nusselt number reduces the magnetic parameter or radiation parameter and increases with the viscosity parameter.

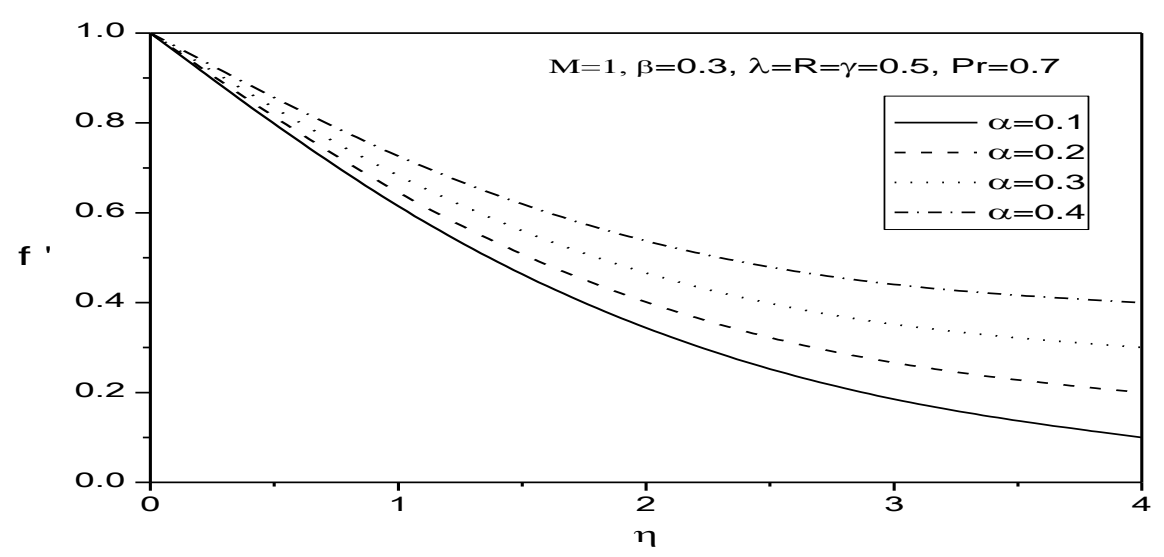

Fig.1 Velocity profiles for different values of $\alpha$ 


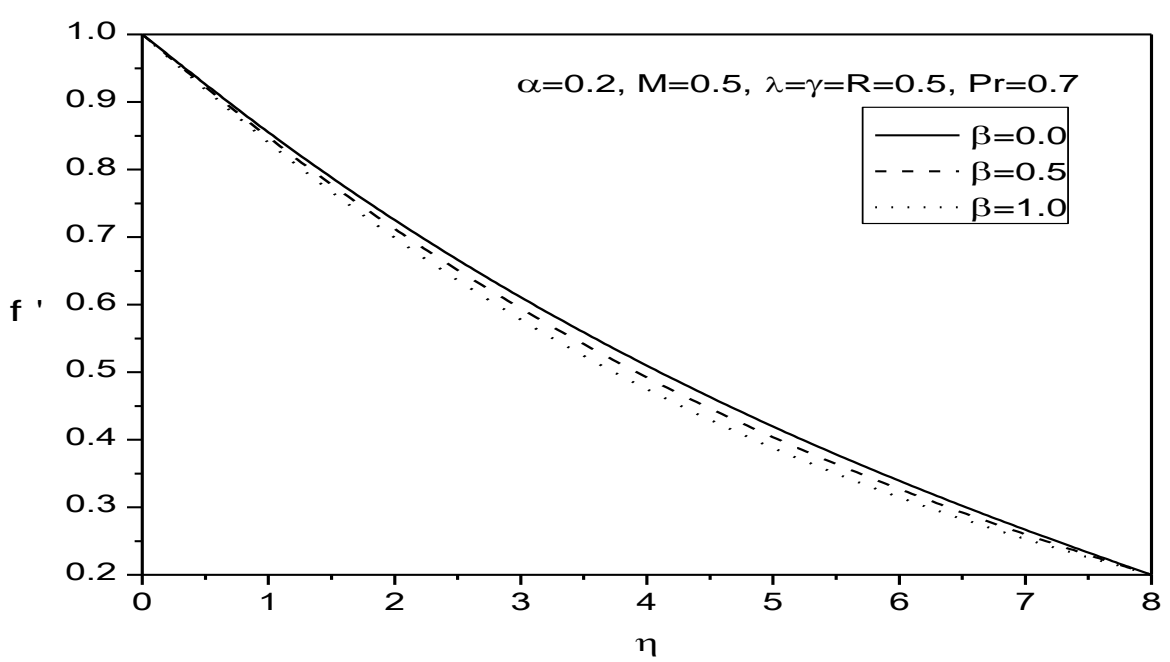

Fig.2 Velocity profiles for different values of $\beta$

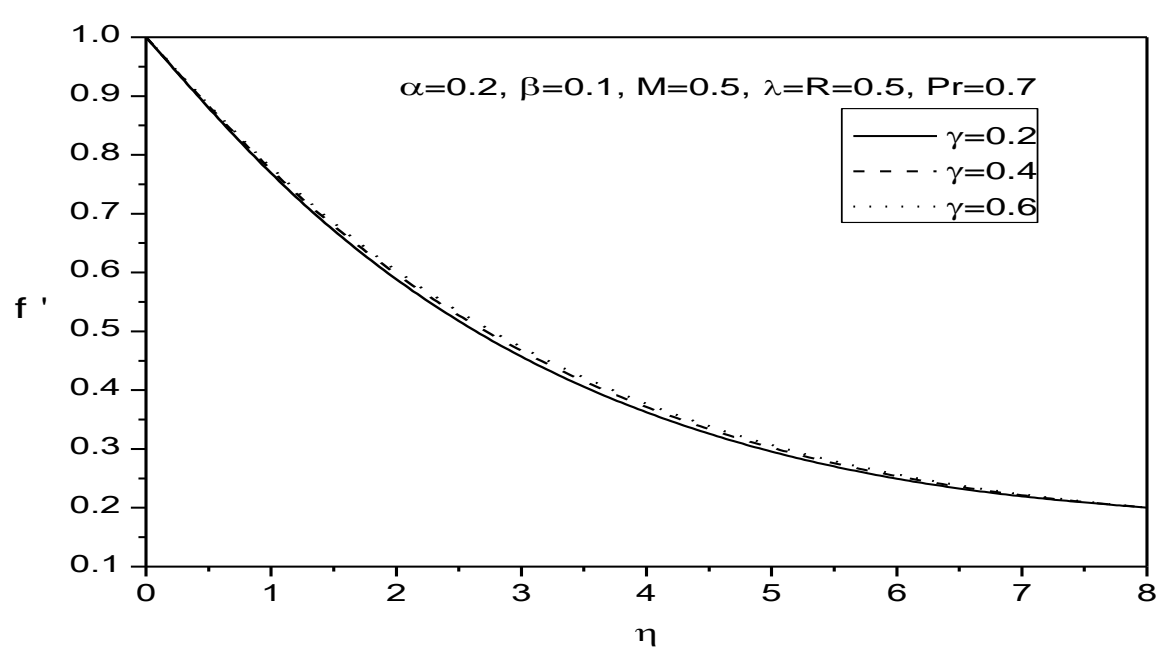

Fig.3 Velocity profiles for different values of $\gamma$

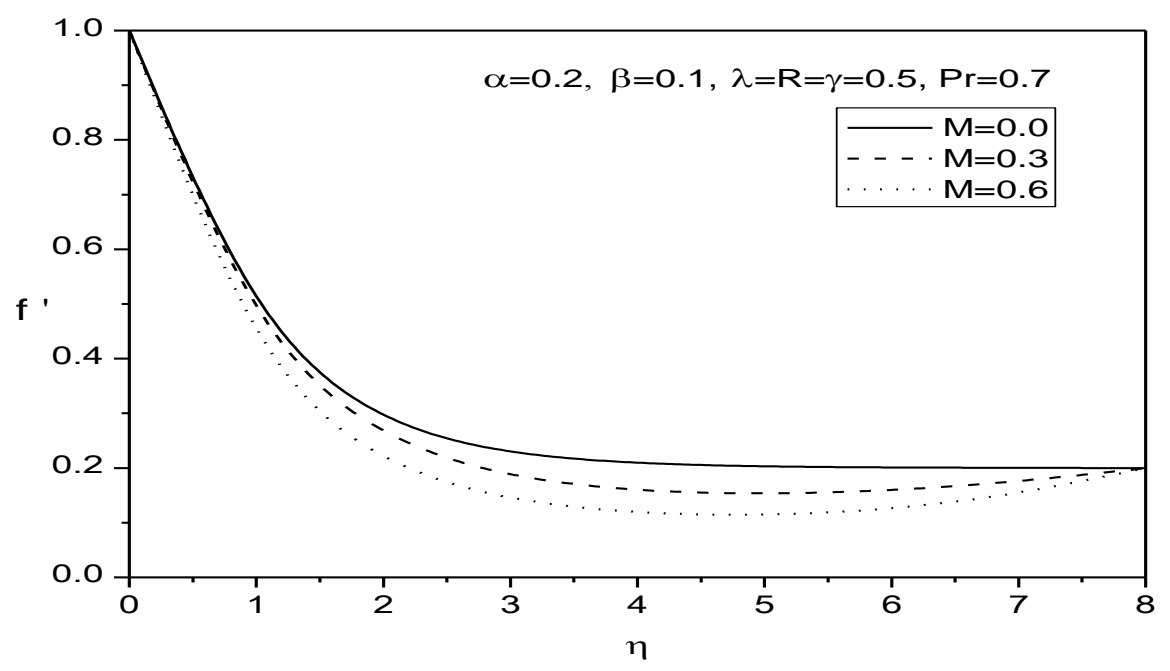

Fig.4 Velocity profiles for different values of $M$ 


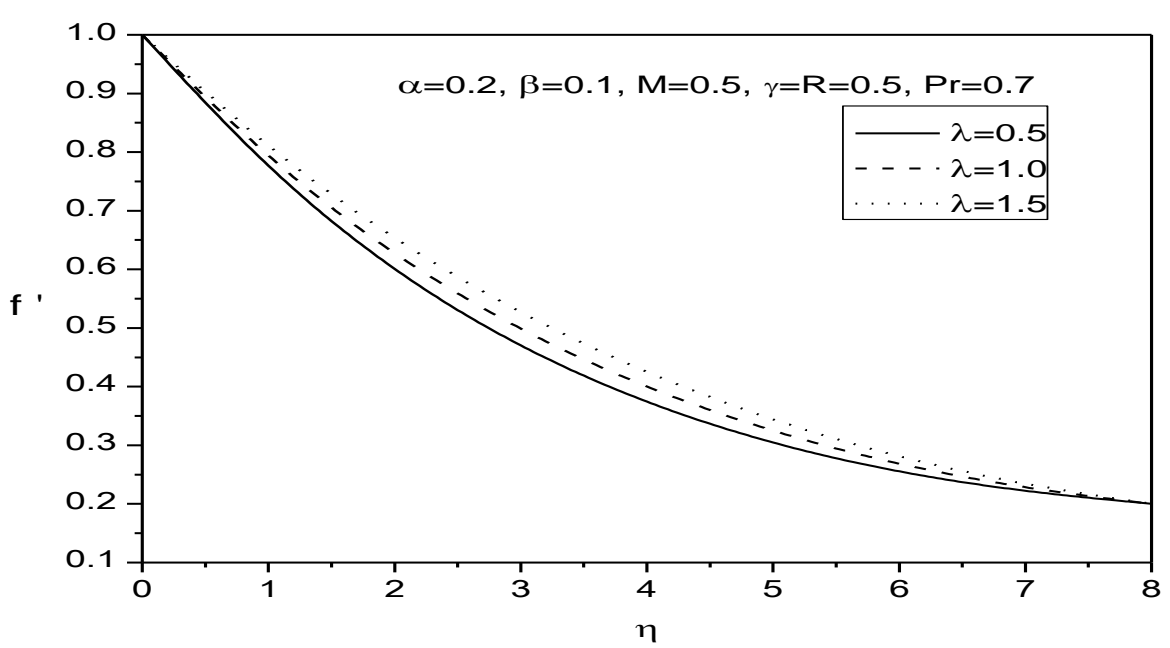

Fig.5 Velocity profiles for different values of $\lambda$

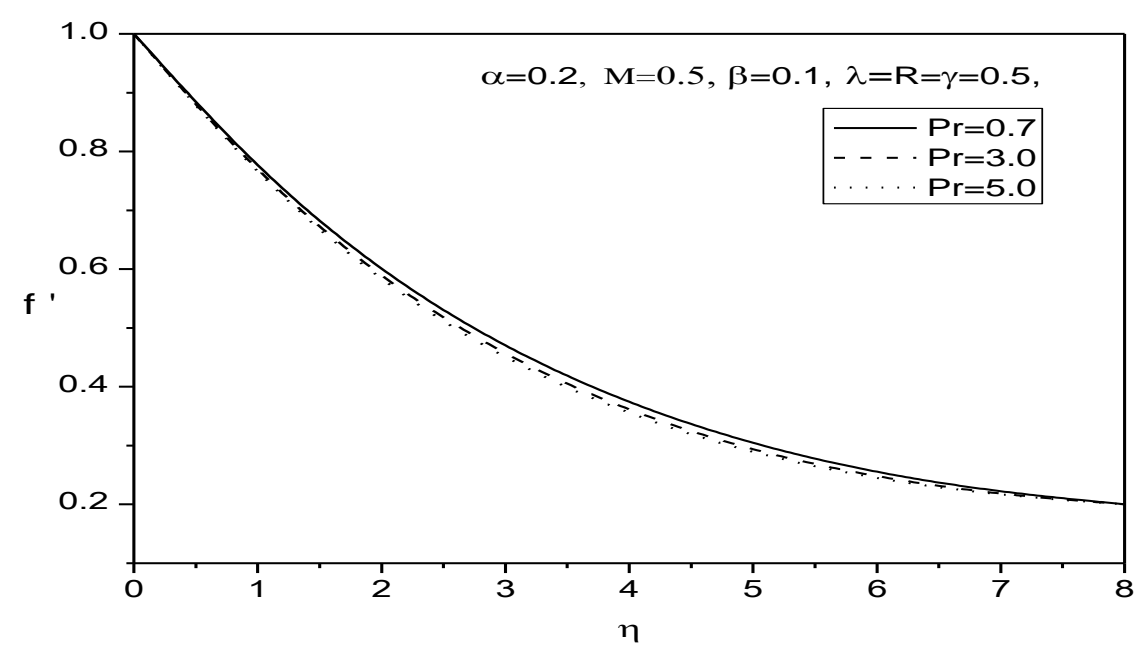

Fig.6 Velocity for different values of $\mathrm{Pr}$

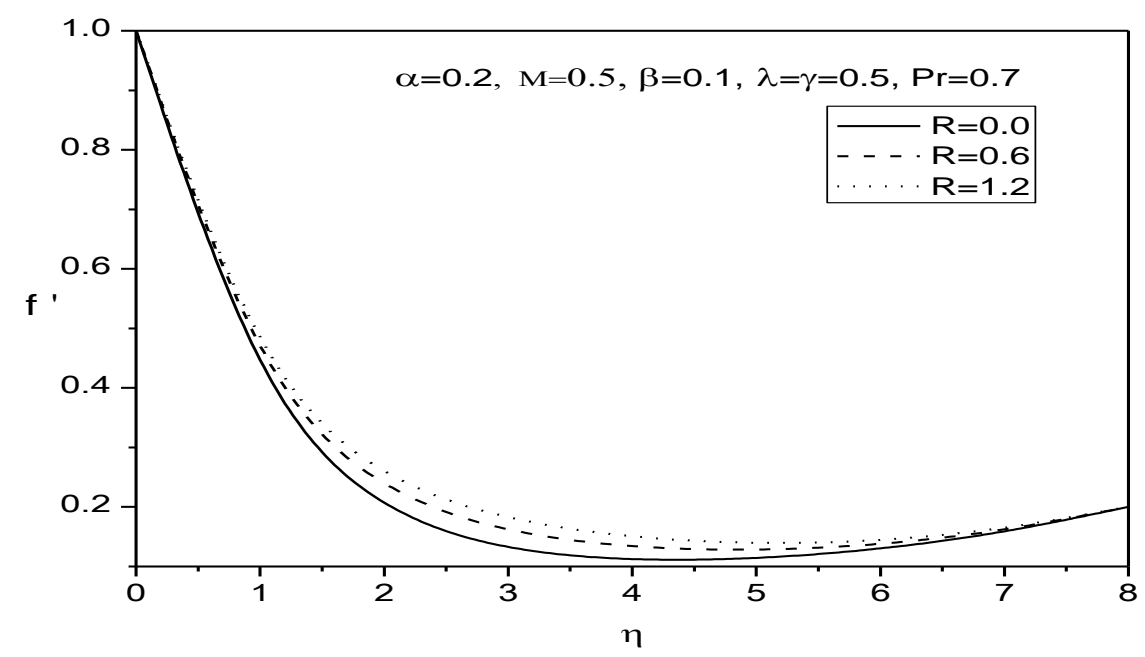

Fig.7 Velocity profiles for different values of $R$ 


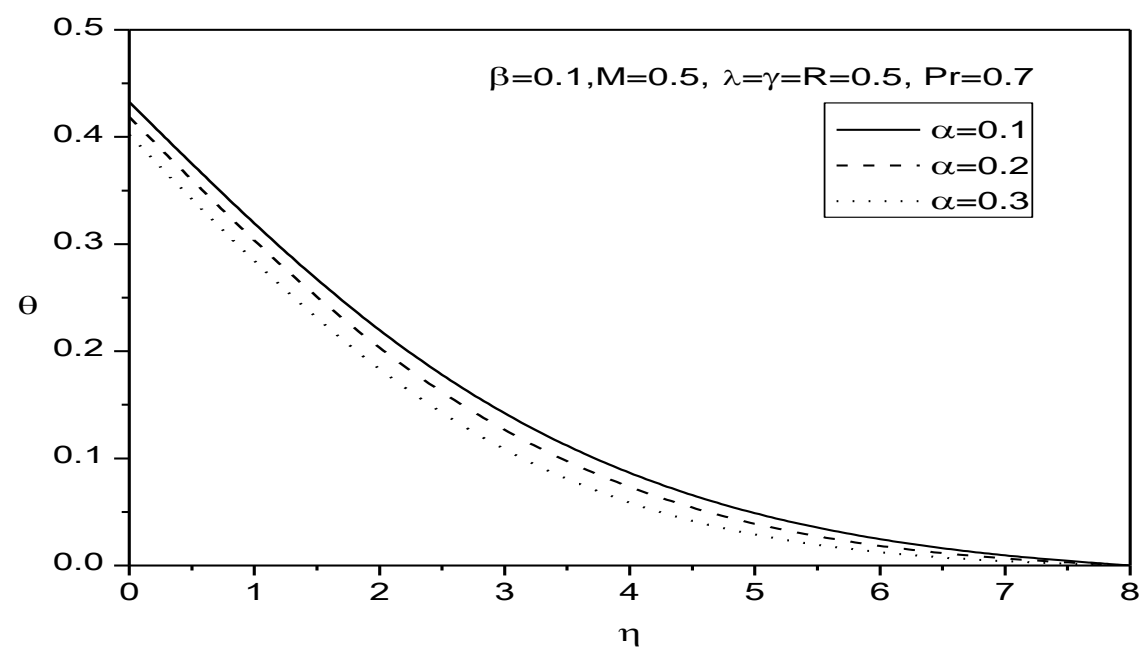

Fig.8 Temperature for different values of $\alpha$

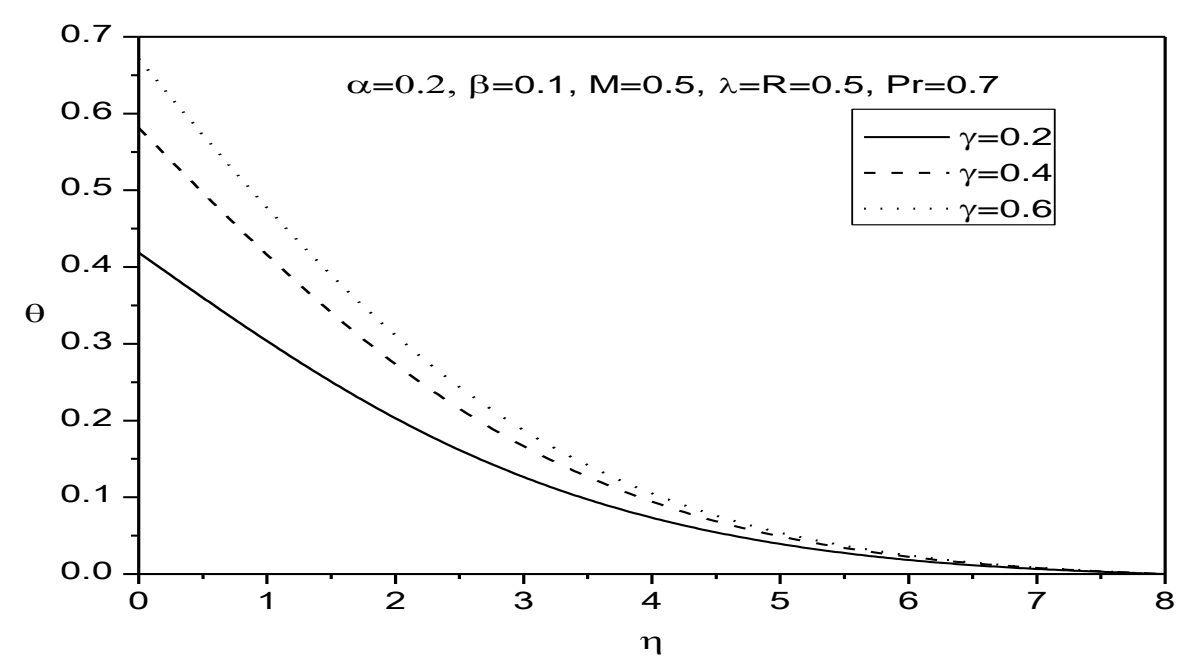

Fig.9 Temperature for different values of $\gamma$

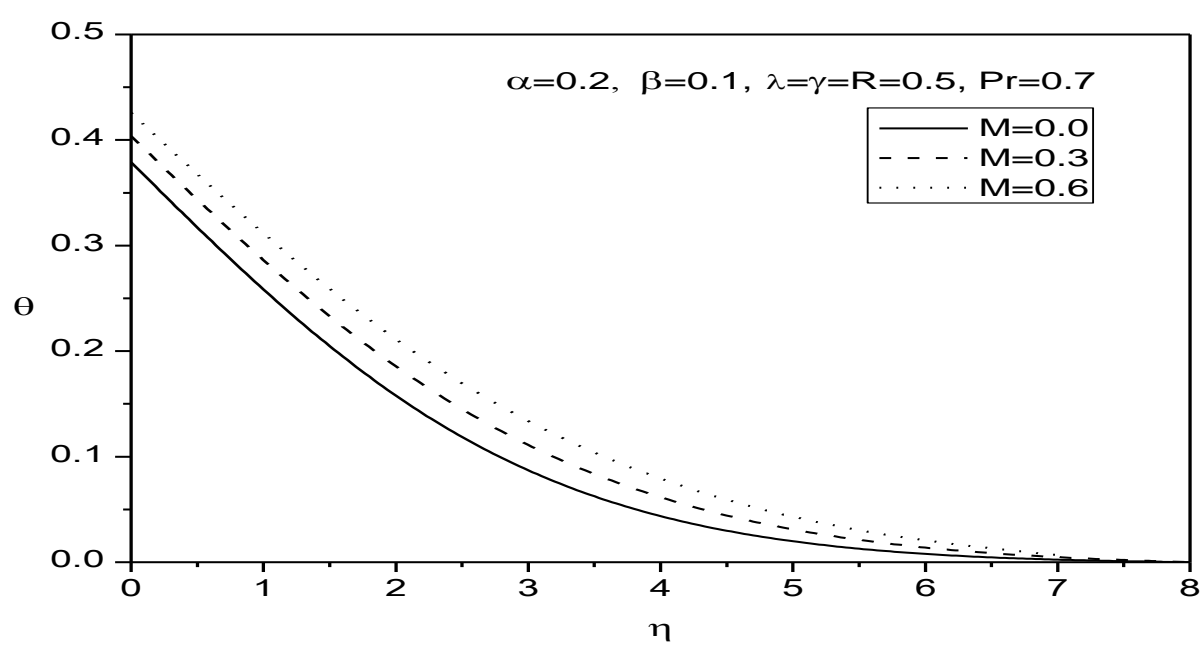

Fig.10 Temperature for different values of $M$ 


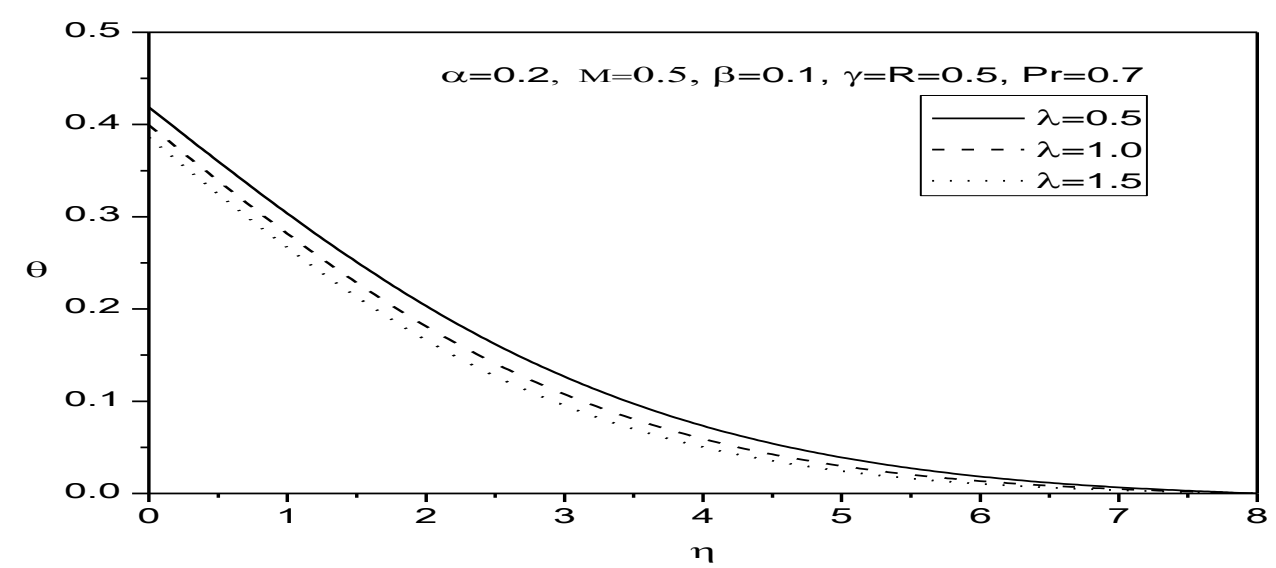

Fig.11 Temperature for different values of $\lambda$

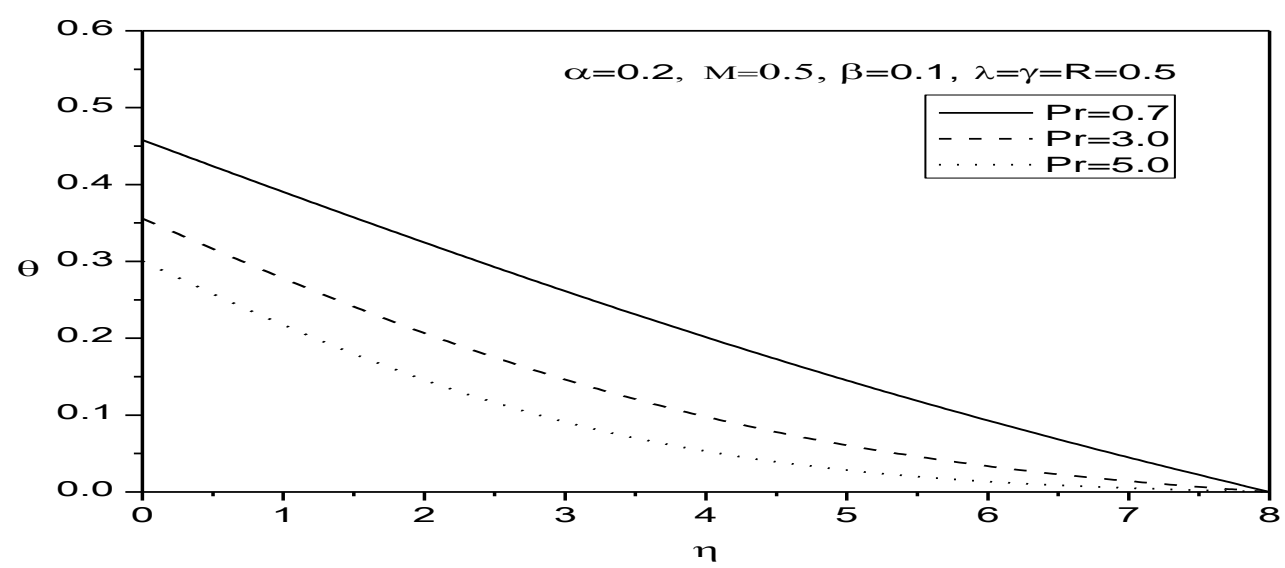

Fig.12 Temperature for different values of $P r$

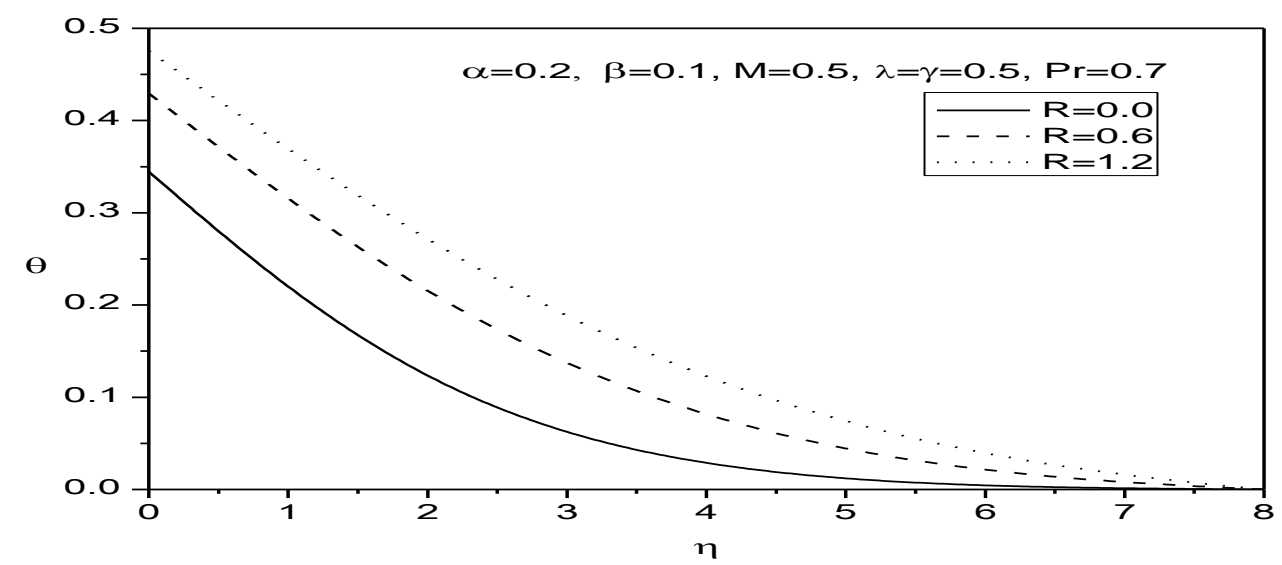

Fig.13 Temperature for different values of $R$

Table 1 Numerical values of $-f^{\prime \prime}(0),-\theta^{\prime}(0)$ at the sheet for different values of $\alpha, \beta, \gamma, M, \lambda, R$ and $P r$.

\begin{tabular}{|c|c|c|c|c|c|c|c|c|}
\hline$\alpha$ & $\beta$ & $\gamma$ & $M$ & $\lambda$ & $R$ & $\operatorname{Pr}$ & $-f^{\prime \prime}(0)$ & $-\theta^{\prime}(0)$ \\
\hline 0.0 & 0.3 & 0.4 & 0.5 & 0.5 & 1.0 & 1.0 & 1.47521 & 0.289624 \\
0.1 & 0.3 & 0.4 & 0.5 & 0.5 & 1.0 & 1.0 & 1.38463 & 0.289749 \\
0.2 & 0.3 & 0.4 & 0.5 & 0.5 & 1.0 & 1.0 & 1.28542 & 0.289881 \\
\hline 0.2 & 0.0 & 0.4 & 0.5 & 0.5 & 1.0 & 1.0 & 1.24794 & 0.289915 \\
0.2 & 0.1 & 0.4 & 0.5 & 0.5 & 1.0 & 1.0 & 1.26033 & 0.289904 \\
0.2 & 0.2 & 0.4 & 0.5 & 0.5 & 1.0 & 1.0 & 1.27783 & 0.289892 \\
\hline
\end{tabular}




\begin{tabular}{|l|l|l|l|l|l|l|l|l|}
\hline 0.2 & 0.3 & 0.1 & 0.5 & 0.5 & 1.0 & 1.0 & 1.31293 & 0.091325 \\
0.2 & 0.3 & 0.4 & 0.5 & 0.5 & 1.0 & 1.0 & 1.28542 & 0.289881 \\
0.2 & 0.3 & 0.8 & 0.5 & 0.5 & 1.0 & 1.0 & 1.26260 & 0.454644 \\
\hline 0.2 & 0.3 & 0.4 & 0.0 & 0.5 & 1.0 & 1.0 & 1.13371 & 0.289981 \\
0.2 & 0.3 & 0.4 & 0.5 & 0.5 & 1.0 & 1.0 & 1.28542 & 0.289881 \\
0.2 & 0.3 & 0.4 & 1.0 & 0.5 & 1.0 & 1.0 & 1.42935 & 0.289787 \\
\hline 0.2 & 0.3 & 0.4 & 0.5 & 0.0 & 1.0 & 1.0 & 1.32560 & 0.289855 \\
0.2 & 0.3 & 0.4 & 0.5 & 0.5 & 1.0 & 1.0 & 1.28542 & 0.289881 \\
0.2 & 0.3 & 0.4 & 0.5 & 1.0 & 1.0 & 1.0 & 1.24529 & 0.289906 \\
\hline 0.2 & 0.3 & 0.4 & 0.5 & 0.5 & 0.0 & 1.0 & 1.28817 & 0.295061 \\
0.2 & 0.3 & 0.4 & 0.5 & 0.5 & 1.0 & 1.0 & 1.28542 & 0.289881 \\
0.2 & 0.3 & 0.4 & 0.5 & 0.5 & 2.0 & 1.0 & 1.28462 & 0.288394 \\
\hline 0.2 & 0.3 & 0.4 & 0.5 & 0.5 & 1.0 & 3.0 & 1.28946 & 0.297499 \\
0.2 & 0.3 & 0.4 & 0.5 & 0.5 & 1.0 & 5.0 & 1.29299 & 0.304245 \\
0.2 & 0.3 & 0.4 & 0.5 & 0.5 & 1.0 & 7.0 & 1.29607 & 0.310218 \\
\hline
\end{tabular}

Table 2 Numerical values of $f^{\prime \prime}(0)$ at the sheet for different values of $\lambda$, Comparison of the present results with that of Pop et al. [23], Mahapatra and Gupta [24] and Hayet et al.[20]

\begin{tabular}{|c|c|c|c|c|}
\hline$\lambda$ & Present study & Pop et al. [23] & Mahapatra and Gupta [24] & Hayet et al.[20] \\
\hline 0.1 & -0.969656 & -0.9694 & -0.9694 & -0.969386 \\
0.2 & -0.918165 & -0.9181 & -0.9181 & -0.918107 \\
0.5 & -0.667686 & -0.6673 & -0.6673 & -0.667264 \\
2.0 & 2.075470 & 2.0174 & 2.0175 & 2.01878 \\
3.0 & 4.731210 & 4.7290 & 4.7293 & 4.72954 \\
\hline
\end{tabular}

\section{References}

[1]. Wang, S. and Tan, W. C., (2011), "Stability Analysis of Soret-Driven Double-Diffusive Convection of Maxwell Fluid in a Porous Medium," International Journal of Heat and Fluid Flow, Vol.32, pp. 88-94.

[2]. Jamil, M. and Fetecau, C., (2010), "Helical Flows of Maxwell Fluid Between Coaxial Cylinders with Given Shear Stresses on the Boundary," Nonlinear Analysis: Real World Applications, Vol.5, pp. 4302-4311.

[3]. Zierep, J. and Fetecau, C., (2007), "Energetic Balance for the Rayleigh-Stokes Problem of a Maxwell Fluid," International Journal of Engineering Science, Vol.45, pp. 617-627.

[4]. Sadeghy, K., Hajibeygi, H. and Taghavi, S. M., (2006), "Stagnation-Point Flow of Upper-Convected Maxwell Fluids," International Journal of Non-Linear Mechanics, Vol.41, pp. 1242-1247.

[5]. Megahed A M 2011 Eur. Phys. J. Plus 126 82, 094701-6

[6]. Yao, S., Fang, T. and Zhong, Y., (2009), "Heat Transfer of a Generalized Stretching/Shrinking Wall Problem with Convective Boundary Conditions," Communications in Nonlinear Science and Numerical Simulation, Vol.14, pp. 154-159.

[7]. Crane, L. J., (1970), Z. Angew. Math. Phys. 21645

[8]. Gupta, P. S., and Gupta, A. S., (1977), Can. J. Chem. Eng. 55744

[9]. Banks, W. H. H., (1983), J. Mec. Theor. Appl. 2375

[10]. Sakiadis, B. C., (1961), Boundary layer behaviour on continuous solid surface: I. Boundary layer equations for two dimensional and axisymmetric flow, AIChE J. Vol.7, pp.26-28.

[11]. Aziz, A., (2009), "A similarity Solution for Laminar Thermal Boundary Layer over a Flat Plate with a Convective Surface Boundary Condition," Communications in Nonlinear Science and Numerical Simulation, Vol.14, pp. 1064-1068.

[12]. Makinde, O. D. and Aziz, A., (2010), "MHD Mixed Convection from a Vertical Plate Embedded in a Porous Medium with a Convective Boundary Condition," International Journal of Thermal Sciences, Vol.49, pp. 1813-1820.

[13]. El-Aziz, M. A., (2010), Meccanica 4597

[14]. Bataller, R. C., (2011), Magnetohydrodynamic Flow and Heat Transfer of an Upper-Convected Maxwell Fluid Due to a Stretching Sheet, FDMP, Vol.7, no.2, pp.153-173.

[15]. Habibi Matin, M., Dehsara, M., Abbassi, A., (2012), Mixed convection MHD flow of nanofluid over a non-linear stretching sheet with effects of viscous dissipation and variable magnetic field, MECHANIKA, Vol. 18(4), pp.415-423.

[16]. Qi, H. and Xu, M., (2007), "Unsteady Flow of Viscoelastic Fluid with Fractional Maxwell Model in a Channel," Mechanics Research Communications, Vol.34, pp. 210-212.

[17]. Abdul Hakeem, A.K., \& Sathiyanathan, K. (2009). An analytic solution of an oscillatory flow through a porous medium with radiation effect, Nonlinear Analysis:Hybrid Systems, Vol.3, pp. 288-295.

[18]. Seddeek, M.A., and Abdelmeguid, M.S., (2006). Effects of radiation and thermal diffusivity on heat transfer over a stretching surface with variable heat flux. Physics Letter A, 348, pp. 172-179.

[19]. Mamun Molla, M., and Anwar Hossain, M., (2007), Radiation effect on mixed convection laminar flow along a vertical wavy surface. International Journal of Thermal Sciences, 46, pp. 926-935.

[20]. Hayat, T., Waqas, M., Shehzad, S. A. and Alsaedi, A., (2013), Mixed Convection Radiative Flow of Maxwell Fluid near a Stagnation Point with Convective Condition, Journal of Mechanics, Vol. 29, No. 3.

[21]. Siti Khuzaimah Soid, Zanariah Mohd Yusof, Ahmad Sukri Abd Aziz, Seripah Awang Kechil, (2012), Magnetohydrodynamics Boundary Layer Flows over a Stretching Surface with Radiation Effect and Embedded in Porous Medium, World Academy of Science, Engineering and Technology, Vol.68,

[22]. Jain, M.K., Iyengar, S.R.K. and Jain, R.K., (1985), Numerical Methods for Scientific and Engineering Computation, Wiley Eastern Ltd., New Delhi, India.

[23]. Pop, S. R., Grosan, T. and Pop, I., (2004), "Radiation Effect on the Flow Near the Stagnation Point of a Stretching Sheet," Technische Mechanik, Vol.25, pp. 100-106.

[24]. Mahapatra, T. R. and Gupta, A. S., (2002), "Heat Transfer in Stagnation-Point Flow Towards a Stretching Sheet," Heat and Mass Transfer, Vol.38, pp. 517-521. 